\title{
Politique
}

\section{La construction de solidarités nationales contre le mouvement syndical}

\section{Raymond Hudon}

Numéro 4, automne 1983

Crise et changements idéologiques

URI : https://id.erudit.org/iderudit/041004ar

DOI : https://doi.org/10.7202/041004ar

Aller au sommaire du numéro

Éditeur(s)

Société québécoise de science politique

ISSN

0711-608X (imprimé)

1918-6584 (numérique)

Découvrir la revue

Citer cet article

Hudon, R. (1983). La construction de solidarités nationales contre le mouvement syndical. Politique, (4), 129-163. https://doi.org/10.7202/041004ar d'utilisation que vous pouvez consulter en ligne.

https://apropos.erudit.org/fr/usagers/politique-dutilisation/ 


\title{
La construction de solidarités nationales contre le mouvement syndical
}

\author{
Raymond Hudon \\ Université Laval
}

Parlant de la crise qui résulte de l'actuel mouvement de restructuration du capitalisme mondial et qui affecte les efforts d'une articulation renouvelée des ensembles économiques «nationaux» à cette réalité changeante, il ressort de plus en plus nettement que cette crise "nous confronte à un formidable redéploiement de la domination du capital sur le travail " ${ }^{1}$. Les justifications les plus élémentaires formulées par divers conseillers de service identifient couramment les taux de rémunération présumément trop élevés accordés aux travailleurs comme une des sources majeures des problèmes aigus qui, largement partagés, sont spécialement sentis au sein des classes populaires. Dans la mesure où l'on parvient à convaincre, les conditions se trouvent ainsi créées pour entreprendre une offensive anti-syndicale parée de l'impératif de construire des solidarités «nationales» assez solides pour permettre de mieux affronter les conditions découlant d'une concurrence internationale implacable.

De ces temps-ci, les interventions gouvernementales rattachées à ce type d'opération se trouvent principalement conju-

1. Vincent Dagenais, "Crise et stratégie syndicale», dans Gilles Dostaler (ed.), La crise économique et sa gestion, Actes du colloque de l'Association d'économie politique (Université du Québec à Montréal, 25-26 septembre 1981), Les Éditions du Boréal Express, Montréal, 1982, 159. 
guées à la nécessité de réduire les déséquilibres budgétaires de l'État et les écarts entre travailleurs du secteur étatique et ceux du secteur privé. De cette manière, les organisations syndicales représentant les travailleuses et les travailleurs du secteur étatique constituent des cibles "privilégiées» pour accomplir une restructuration significative des rapports de forces. Pour dégager le sens des gestes plus récents posés par les gouvernements canadien et québécois et surtout pour saisir leur portée réelle sur le mouvement syndical, il s'impose de dépasser le caractère plus immédiat de ces événements.

Ainsi, cette présentation vise d'abord à cerner les sources de l'offensive anti-syndicale qui se manifeste maintenant avec transparence. Il apparaît pertinent de faire subséquemment ressortir, par une brève présentation de la réponse syndicale, la capacité limitée de résistance qui marque présentement le syndicalisme. En une période où la défense de ses acquis se révèle suspecte pour ceux qui, nombreux, ont déjà tout ou beaucoup perdu, la légitimité de l'action syndicale se trouve profondément minée. C'est dans ce contexte de transformations des pratiques syndicales, pas nécessairement du discours, que prennent place les appels plus pressants des dirigeants politiques en faveur d'un effort national et d'un mode de fonctionnement "corporatiste". Ces appels sont définis plus précisément dans une troisième partie pour justement faire voir leur contribution à un affaiblissement encore plus prononcé des positions syndicales. La prise en considération des divers éléments qui précèdent permet enfin de mettre en perspectives l'effritement observable des solidarités syndicales et de relier ce dernier phénomène à des tendances plus profondes au sein des sociétés contemporaines. 


\section{Les sources de l'offensive anti-syndicale}

Les gains obtenus par les travailleurs le furent régulièrement au prix de luttes âpres et soutenues. La volonté manifestée depuis un peu plus d'une dizaine d'années de réduire le coût du travail ne révèle donc pas des attitudes forcément nouvelles. La détermination démontrée à réaliser cette volonté constitue cependant une des spécificités de la dernière décennie.

Au début des années soixante-dix, le gouvernement canadien fit le pari que les organisations syndicales collaboreraient en restreignant volontairement les revendications salariales de leurs membres pour ainsi assurer la compression de la part des salaires dans le calcul des coûts de production. Les raisons invoquées pour appuyer une telle démarche étaient généralement ramenées à l'impérieuse nécessité de juguler l'inflation à laquelle on attribuait des effets néfastes sur la capacité concurrentielle de l'industrie canadienne au sein du marché mondial. Comme l'illustre ce témoignage de John Deutsch à la fin de la deuxième Conférence économique nationale tenue sous sa présidence en 1974 , on ne rejeta pas le recours aux menaces à peine voilées pour convaincre d'une adhésion volontaire aux principes de restrictions :

Tenons-nous à notre genre de société avec ses libertés, ses potentialités, sa flexibilité et ses possibilités de progrès sociaux? Ou, au contraire, en arriverons-nous à un autre système social ou politique qui sera l'aboutissement d'un taux continu et toujours plus élevé d'inflation? Un tel taux continu et toujours plus élevé d'inflation produirait des contraintes et des changements sociaux ainsi que des tensions auxquels nous serions incapables de parer, sauf par l'utilisation de processus différents et plus arbitraires que ceux auxquels nous sommes habitués. ${ }^{2}$

Plutôt que d'accepter si «aimable» invitation à la modération, les organisations syndicales réclamèrent avec ardeur l'inde-

2. Conseil économique du Canada, Évolution des priorités. Compte rendu de la Conférence économique nationale, (Montréal, $1^{\mathrm{er}}-3$ décembre 1974), Information Canada, Ottawa, 1975, 64. 
xation des salaires de leurs membres. Ces réclamations furent spécialement vigoureuses au Québec où il fut décidé «de lancer une vaste campagne en vue de la réouverture des conventions collectives et des décrets » en s'appuyant sur le précédent créé en 1972 par l'obtention de cette indexation pour les travailleurs des services publics alors réunis en Front commun. ${ }^{3}$ Devant la détermination affichée par les travailleurs, on dut faire bon nombre de concessions. Mais ce fut bien à contrecour. Car l'aggravation de la récession empêchait, comme le souligne Cy Gonick, que l'on refile aux consommateurs l'accroissement des coûts du travail. Le résultat fut que la progression des profits des compagnies entre 1974 et 1975 connut un déclin sensible. ${ }^{4}$ Une intervention énergique apparut donc s'imposer.

En octobre 1975, le gouvernement Trudeau imposa le contrôle des prix et des salaires malgré qu'il ait fait campagne en 1974 en s'opposant spécifiquement au projet conservateur en ce sens. L'effet recherché fut en grande partie atteint, comme l'a noté le Conseil économique du Canada (C.E.C.) qu'on peut difficilement accuser d'être à la remorque du discours syndical: «Les résultats obtenus avec ce programme indiquent que l'application de mesures de contrôle pendant quelques années peut contribuer à ralentir considérablement le rythme d'augmentation des salaires ». ${ }^{5}$ Voyons plus précisément à quoi correspondent les résultats obtenus.

Sous l'effet des contrôles, la hausse salariale moyenne consentie pour la première année dans les principales conventions collectives est tombée

3. (Collectif), 150 ans de luttes. Histoire du mouvement ouvrier au Québec (18251976), Confédération des syndicats nationaux/Centrale de l'enseignement du Québec, Montréal/Québec, 1979, 193.

4. Cy Gonick, Inflation and Wage Controls, A Canadian Dimension Publication, [s.1.], 1976, 117.

5. Conseil économique du Canada, Les temps diffciles. Politiques et contraintes, Dix-neuvième exposé annuel, Approvisionnements et Services Canada, Ottawa, 1982, 107. 
d'environ $20 \%$ en 1975 à $7 \%$ en 1978. Cette baisse correspond trop étroitement à la ligne directrice alors en vigueur en matière de revenu pour être considérée comme une simple coincidence. Par ailleurs, l'impact décisif du Programme de lutte contre l'inflation sur les ententes salariales a été confirmé par des analyses économétriques.

Le Programme de lutte contre l'inflation n'a pas donné d'aussi bons résultats dans le cas de l'augmentation des prix. L'IPC [indice des prix à la consommation] n'a enregistré qu'une faible baisse, passant d'un peu moins de $11 \%$ en 1975 à $9 \%$ en 1978. Cela représente plus du double de l'objectif de $4 \%$ prévu dans le Livre blanc intitulé Offensive contre l'inflation, qui fut rendu public au moment de la mise en ouvre du programme. ${ }^{6}$

Comme le fait remarquer le C.E.C., ces résultats «ont confirmé les soupçons répandus dans le mouvement ouvrier à l'effet que ces mesures [de contrôle] ne visent pas tant les prix que les salaires. Par conséquent, les milieux ouvriers s'opposent plus que jamais à ce genre de réglementation, et cette opposition se manifeste aussi à l'égard d'autres formes de politiques de revenus. » Cette opposition se trouve cependant en grande partie limitée par le fait que "les contrôles jouissent d'un appui considérable parmi le public en général ". ${ }^{7}$ De plus, l'état de mobilisation des forces syndicales, dont il sera plus longuement question ultérieurement, n'est plus actuellement ce qu'il était il y a sept ou huit ans; on ne pourrait vraisemblablement répéter qu'avec grande difficulté une journée de grève générale contre un éventuel contrôle des salaires comme celle qui, le 14 octobre 1976, avait rallié " 1.2 million de syndiqués canadiens" 8 .

Pourtant, les menaces de recours à des contrôles se révèlent aujourd'hui plus explicites que lors de la période qui avait précédé les contrôles de 1975. Le C.E.C. exprime ainsi l'avis net qu'une politique visant «les hausses de prix et de salaires» risque de demeurer grandement inefficace «aussi longtemps que la

6. Ibid., 108

7. Idem.

8. (Collectif), 150 ans de luttes, op. cit., 195. 
politique conserve un caractère volontaire et qu'elle ne prévoit aucune sanction, [car] il demeure difficile de convaincre les particuliers et les groupes de sacrifier leurs propres intérêts en se privant d'un revenu plus élevé pour le bien général, ce dernier prenant la forme d'une baisse de l'inflation $» .{ }^{9}$ La défense des intérêts particuliers des membres qui fonde l'existence de l'organisation syndicale fournit toute la matière utile pour justifier une attaque énergique contre le mouvement syndical, en prenant exemple sur l'administration Reagan: «Les mesures rigoureuses prises à l'égard des contrôleurs aériens ont permis au gouvernement américain de manifester sans équivoque son intention de ne pas déroger des lignes directrices, même sous la menace de grèves illégales. » 10

Le lien direct établi entre une hausse des salaires et la progression de l'inflation a souvent été contesté par les porte-parole syndicaux. Les tenants d'un programme de contrôles reconnaissent parfois que l'incidence des salaires sur le taux d'inflation n'est peut-être pas si important, précisant même que le revenu réel des travailleurs peut avoir connu un déclin "1. Malgré les acquis indiscutables des politiques de restriction des hausses salariales illustrées par les données du tableau $1,{ }^{12}$ il semble bien qu'on juge les résultats obtenus encore très insuffisants, comme le montrent les commentaires formulés par le C.E.C.

[Le tableau 1] permet de voir comment l'effet conjugué de l'évolution des taux de change et des fluctuations dans les taux de rémunération de

9. Conseil économique du Canada, Les temps difficiles, ap. cit., 107. Ce type d'argument n'est aucunement réservé aux membres du C.E.C.; voir, par exemple, Clarence L. Barber et John C.P. McCallum, Controlling Inflation. Learning from Experience in Canada, Europe and Japan, Institut canadien de politique économique/James Lorimer \& Company, Publishers, Ottawa/Toronto, 1982, 12.

10. Conseil économique du Canada, Les temps diffuiles, op. cit., 107. 99- 100.

11. Clarence L. Barber et John C. P. McCallum, Controlling Inflation, op. cit., cit., 62 .

12. Tableau reproduit de Conseil économique du Canada, Les temps diffriles, op. 


\section{TABLEAU}

Rémunération horaire estimative ' des travailleurs de la production dans l'industrie manufacturière,

Canada et autres pays industrialisés, 1970-1981

Dollars É.-U.

\begin{tabular}{|c|c|c|c|c|c|c|c|c|c|c|c|c|}
\hline & 1970 & 1971 & 1972 & 1973 & 1974 & 1975 & 1976 & 1977 & 1978 & 1979 & 1980 & 1981 \\
\hline \multicolumn{13}{|c|}{ Pays industrialisés: } \\
\hline Canada & 3,46 & 3,91 & 4,31 & 4,66 & 5,45 & 6,11 & 7,20 & 7,54 & 7,69 & 8,15 & 9,04 & 9,86 \\
\hline États-Unis & 4,18 & 4,49 & 4,84 & 5,26 & 5,75 & 6,35 & 6,93 & 7,59 & 8,30 & 9,08 & 10,00 & 11,06 \\
\hline Belgique & 2,06 & 2,44 & 3,16 & 4,19 & 5,12 & 6,54 & 7,03 & 8,46 & 10,39 & 12,02 & 13,18 & 11,13 \\
\hline France & 1,72 & 1,93 & 2,34 & 3,08 & 3,41 & 4,58 & 4,76 & 5,31 & 6,54 & 7,90 & 9,23 & 8,28 \\
\hline Allemagne & 2,33 & 2,76 & 3,34 & 4,56 & 5,32 & 6,19 & 6,60 & 7,79 & 9,65 & 11,26 & 12,26 & 10,47 \\
\hline Italie & 1,74 & 2,10 & 2,55 & 3,17 & 3,62 & 4,60 & 4,38 & 5,08 & 6,09 & 7,19 & 8,26 & 7,59 \\
\hline Pays-Bas & 2,12 & 2,55 & 3,13 & 4,28 & 5,33 & 6,53 & 6,98 & 8,15 & 9,98 & 11,47 & 12,17 & 10,25 \\
\hline Suède & 2,93 & 3,23 & 4,03 & 4,93 & 5,63 & 7,18 & 8,21 & 8,85 & 9,65 & 11,33 & 12,51 & 11,88 \\
\hline Royaume-Uni & 1,49 & 1,74 & 2,03 & 2,25 & 2,60 & 3,27 & 3,12 & 3,35 & 4,28 & 5,50 & 7,37 & 7,43 \\
\hline Japon & 0,99 & 1,18 & 1,58 & 2,19 & 2,67 & 3,05 & 3,30 & 4,03 & 5,54 & 5,49 & 5,61 & 6,23 \\
\hline \multicolumn{13}{|c|}{$\begin{array}{l}\text { Pays nouvellement } \\
\text { industrialisés : }\end{array}$} \\
\hline Mexique & $"$ & $"$ & $"$ & $"$ & $n$ & 1,89 & 1,95 & 1,75 & 2,01 & 2,34 & 2,97 & 3,65 \\
\hline Brésil & $"$ & $"$ & $"$ & $"$ & $"$ & 1,13 & 1,29 & 1,46 & 1,67 & 1,73 & 1,70 & 2,14 \\
\hline Corée & $"$ & $"$ & $"$ & $"$ & $"$ & 0,37 & 0,47 & 0,63 & 0,86 & 1,14 & 1,09 & 1,17 \\
\hline Hong Kong & $"$ & $"$ & $"$ & $"$ & $"$ & $"$ & $"$ & $"$ & 1,13 & 1,25 & 1,30 & 1,40 \\
\hline Tä̈wan & $"$ & $"$ & $"$ & $"$ & $"$ & $"$ & $"$ & $"$ & 0,80 & 1,01 & 1,27 & 1,52 \\
\hline
\end{tabular}

${ }^{1}$ La rémunération horaire totale comprend tous les paiements directs versés au travailleur (rémunération pour le travail effectué, les vacances et autres congés rémunérés, et toute rémunération en nature ou sous forme de primes, avant toute retenue sur le salaire, quelle qu'elle soit) Elle comprend également les «avantages sociaux» comme les déboursés de l'employeur au titre de la sécurité sociale, des assurances, etc. Ces données sont tirées d'enquêtes périodiques portant sur les coûts de main-d'œuvre, les résultats ont été interpolés pour les années intermédiaires. Les petites différences entre les niveaux de rémunération ne doivent pas être considérées comme significatives. La rémunération totale est calculée par heure de travail.

Source: U.S. Department of Labor. Bureau of Labor Statistics, Office et Productivity and Technology, juillet 1982 (données inédites). 
la main-d'ouvre a favorisé le Canada au cours de la dernière décennie. Alors qu'au début des années 70 , le salaire horaire au Canada venait en seconde place derrière les États-Unis, à la fin de la décennie, la rémunération salariale (exprimée en dollars E.-U.) dans six des dix pays industrialisés était plus élevée qu'au Canada. Par contre, les coûts de fabrication au Japon représentent moins des deux tiers de ceux enregistrés au Canada. Il est encore plus frappant de constater que les niveaux de rémunération dans les pays en voie de développement les plus avancés ne représentent qu'entre un huitième et un tiers de ceux versés au $\mathrm{Ca}$ nada ${ }^{13}$.

Les préoccupations qui guident et appuient la volonté manifestée de réduire le taux de croissance de la rémunération dépassent donc visiblement l'objectif apparemment plus concentré d'une politique de réduction du rythme de croissance de l'inflation. Elles doivent être aussi rattachées aux exigences de la concurrence internationale, comme le font directement ressortir les commentaires du C.E.C. qui précise: «Les données sur les niveaux de rémunération mettent en relief l'avantage concurrentiel dont disposent les pays nouvellement industrialisés, surtout dans le cas des produits dont la technologie est largement accessible $^{14}$.» Il faut cependant ajouter que les employeurs ne se limitent pas à téclamer une décélération du rythme de croissance des salaires. Le Conseil du patronat du Québec (C.P.Q.) n'avait-il pas, au milieu des années soixante-dix, soutenu que les salariés occupaient «sans doute la meilleure situation de tous les partenaires sociaux» (sic) en référant à des avantages débordant la comptabilisation des seuls salaires?

Avec le développement industriel, l'employé a vu diminuer son insécurité économique. Non seulement l'entreprise industrielle moderne offre-t-elle de bons salaires mais les profits qui assurent sa croissance garantissent aussi à ses employés une relative sécurité d'emploi. De plus, elle offre une foule d'avantages complémentaires, des bénéfices qui sont de moins en moins «marginaux». Une récente étude du Thorn

13. Idem.

14. Idem. 
Group de Toronto portant sur cent compagnies privées confirme que l'ensemble des bénéfices marginaux, qui n'occupaient en 1953 que $15 \%$ de la masse salariale, représentent aujourd'hui $28 \%$ de cette même masse salariale. ${ }^{15}$

Cette évaluation du C.P.Q. n'est pas tout à fait erronée. Il importe tout de même de la compléter, en autant que le Canada est concerné, par une comparaison avec ce qui s'est passé dans d'autres pays industrialisés pour ainsi placer dans une juste perspective ce que l'on peut observer touchant l'accroissement des bénéfices additionnels à la rémunération horaire directe des travailleurs de la fabrication au Canada. Les données présentées au tableau $2^{16}$ conduisent d'ailleurs les analystes de l'Agence d'examen de l'investissement étranger à y voir des avantages remarquables pour les employeurs canadiens.

Les bénéfices additionnels à la rémunération horaire directe ainsi que le taux de change s'avèrent les plus importants facteurs de l'écart entre le niveau de rémunération au Canada et celui des autres pays industrialisés. Selon [une étude récente du ministère de l'Industrie et du Commerce], le taux des bénéfices additionnels à la rémunération horaire dans le secteur canadien de la fabrication était de 30 pourcent en 1980, alors qu'il atteignait 36 pourcent aux États-Unis et s'échelonnait entre 63 et 97 pourcent en Allemagne, en Belgique, en France, en Italie, en Suède et aux Pays-Bas. Ainsi, les employeurs canadiens ont l'avantage d'une charge de rémunération moins lourde au chapitre de bénéfices que la plupart des autres pays industrialisés ${ }^{17}$

Ces données, éloquentes en elles-mêmes, prennent une signification encore plus importante quand on ajoute que «ces comparaisons ne tiennent pas compte des niveaux de productivité, qui sont importants dans le calcul des coûts de maind'œuvre» et surtout quand on précise que «la productivité au Canada, définie en termes de production par personne-heure,

15. Conseil du patronat du Québec, Des profits, oui, mais pour qui? , Les Éditions du Jour, Montréal, 1976, 45.

16. Tableau tiré de L'investisseur étranger, vol. 5, no. 1, automne 1981, 3.

17. «Niveau de rémunération assez bas au Canada», dans ibid., 2-3. 
TABLEAU 2

Taux des bénéfices additionnels à la rémunération horaire directe des travailleurs de la fabrication dans dix pays a

\begin{tabular}{lllllll}
\hline & 1960 & 1965 & 1970 & 1975 & 1979 & 1980 \\
\hline Érars-Unis & 17,9 & 20,3 & 24,7 & 31,4 & 35,5 & 36,4 \\
Belgique & 38,9 & 47,9 & 56,2 & 68,3 & 72,1 & 72,4 \\
Canada & 15,4 & 16,2 & 19,8 & 22,8 & 29,2 & 30,1 \\
France & 54,5 & 62,0 & 60,3 & 70,9 & 79,6 & 80,6 \\
Allemagne & 35,0 & 36,5 & 43,1 & 57,3 & 62,3 & 63,4 \\
Italie & 70,3 & 75,9 & 79,2 & 99,4 & 96,0 & 97,0 \\
Pays-Bas & $38,5^{*}$ & $43,8^{*}$ & $56,7^{*}$ & $70,0^{*}$ & $72,1^{*}$ & $73,6^{*}$ \\
Suede & 16,5 & 22,7 & 26,5 & 45,4 & 61,3 & 63,0 \\
R.-U. & 10,9 & 13,3 & 15,0 & 23,9 & 30,0 & 31,7 \\
Japon & $12,6^{*}$ & $13,1^{*}$ & $13,2^{*}$ & $14,3^{*}$ & $18,8^{*}$ & $20,0^{*}$ \\
\hline
\end{tabular}

Note: les données de 1979 sont des estimés préliminaires. Les estimés de 1980 sont provisoires.

* tous les employés

a ces taux sont en devises locales

Source: Données du ministère américain du travail, op. cit., mars $198 \mathrm{l}$.

s'est accrue à un taux moyen annuel de 3,7 pourcent entre 1961 et 1980, soit à un taux beaucoup plus élevé qu'aux ÉtatsUnis ${ }^{18}$.

Ces attaques sur la rémunération globale, non seulement sur la rémunération directe, ne diminuent aucunement la détermination à affaiblir la résistance syndicale, surtout si l'on considère que plusieurs des avantages additionnels à la rémunération directe furent régulièrement obtenus à la suite de pressions syndicales. Ce que les organisations syndicales manquent rarement de rappeler. L'offensive anti-syndicale trouve encore des sources supplémentaires de justification dans l'effet de nivellement ou, plus justement, de réduction des écarts salariaux que l'action de négociation entraîne. Comme le montrent les don- 
nées du tableau $3^{19}$, l'influence des syndicats apparaît sensible, car «l'étude de la distinction entre travailleurs syndiqués et non syndiqués indique que l'action syndicale a contribué à accélérer la compression des écarts, puisque, dans chacun des emplois spécialisés retenus, la différence est nettement plus faible chez les travailleurs syndiqués " 20 .

Dans la mesure où il est juste d'assumer avec le C.E.C. que «les travailleurs n'éprouvent pas d'attrait pour les emplois hautement spécialisés dont le niveau de rémunération ne compense pas le temps supplémentaire affecté à la formation, ce qui mène à des pénuries dans cette catégorie d'emplois " ${ }^{21}$, il se révèle probable que l'on en vienne à percevoir l'action syndicale comme une nuisance. Cette présomption de nuisance syndicale est ostensiblement déduite de l'identification explicite de « toute une gamme de politiques et de programmes touchant les salaires et les heures de travail, la sécurité et l'hygiène industrielles, les griefs et la sécurité d'emploi» ${ }^{22}$. Il serait difficile de trouver une allusion plus directe aux effets de «distorsion» introduits par les syndicats, effets que l'on se propose de contourner par des programmes de création d'emplois!

On trouve, dans le marché du travail d'aujourd'hui, un certain nombre de programmes visant à sauvegarder les droits et le bien-être des travailleurs. Ces mesures perturbent néanmoins l'action des forces purement économiques et peuvent introduire des désincitations et des distorsions dans le processus d'allocation des travailleurs. Certains employeurs considèrent, par exemple, qu'il existe un écart considérable entre la productivité attendue des ouvriers les moins qualifiés et le salaire brut

19. Tableau tiré de Conseil économique du Canada, Pénuries et carences. Travailleurs qualifiés et emplois durant les années 80, Approvisionnements et Services Canada, Ottawa, $1982,78$.

20. Ibid., 77 .

21. Ibid., 75 .

22. Ibid., 3 . 
Écarts de salaire ${ }^{1}$ interprofessionnels pour les travailleurs syndiqués et non syndiqués entre certaines catégories hautement spécialisées et une catégorie non spécialisée, Canada, 1974-1979

Pourcentages

\begin{tabular}{|c|c|c|c|c|c|c|c|}
\hline & Statut syndical ${ }^{2}$ & 1974 & 1975 & 1976 & 1977 & 1978 & 1979 \\
\hline Outilleur-ajusteur & $\begin{array}{l}\text { Syndiqué } \\
\text { Non syndiqué }\end{array}$ & $\begin{array}{l}142,7 \\
153,1\end{array}$ & $\begin{array}{l}132,8 \\
150,6\end{array}$ & $\begin{array}{l}128,8 \\
147,9\end{array}$ & $\begin{array}{l}129,4 \\
152,2\end{array}$ & $\begin{array}{l}132,3 \\
153,2\end{array}$ & $\begin{array}{l}128,7 \\
158,2\end{array}$ \\
\hline Opérateur de machine, entretien & $\begin{array}{l}\text { Syndiqué } \\
\text { Non syndiqué }\end{array}$ & $\begin{array}{l}135,1 \\
144,8\end{array}$ & $\begin{array}{l}129,4 \\
141,5\end{array}$ & $\begin{array}{l}127,3 \\
142,1\end{array}$ & $\begin{array}{l}125,4 \\
141,0\end{array}$ & $\begin{array}{l}128,3 \\
141,3\end{array}$ & $\begin{array}{l}126,5 \\
145,8\end{array}$ \\
\hline Soudeur, entretien & $\begin{array}{l}\text { Syndiqué } \\
\text { Non syndiqué }\end{array}$ & $\begin{array}{l}139,8 \\
145,3\end{array}$ & $\begin{array}{l}132,7 \\
147,9\end{array}$ & $\begin{array}{l}132,1 \\
148,7\end{array}$ & $\begin{array}{l}129,8 \\
144,8\end{array}$ & $\begin{array}{l}132,2 \\
144,3\end{array}$ & $\begin{array}{l}128,3 \\
150,3\end{array}$ \\
\hline $\begin{array}{l}\text { Réparateur d'appareillage } \\
\text { électrique }\end{array}$ & $\begin{array}{l}\text { Syndiqué } \\
\text { Non syndiqué }\end{array}$ & $\begin{array}{l}142,8 \\
161,2\end{array}$ & $\begin{array}{l}136,3 \\
159,4\end{array}$ & $\begin{array}{l}134,0 \\
157,1\end{array}$ & $\begin{array}{l}131,7 \\
154,2\end{array}$ & $\begin{array}{l}135,1 \\
155,3\end{array}$ & $\begin{array}{l}132,3 \\
155,7\end{array}$ \\
\hline Ajusteur-monteur & $\begin{array}{l}\text { Syndiqué } \\
\text { Non syndiqué }\end{array}$ & $\begin{array}{l}142,3 \\
151,9\end{array}$ & $\begin{array}{l}133,7 \\
154,6\end{array}$ & $\begin{array}{l}132,1 \\
154,2\end{array}$ & $\begin{array}{l}130,9 \\
149,2\end{array}$ & $\begin{array}{l}135,2 \\
154,6\end{array}$ & $\begin{array}{l}132,9 \\
150,2\end{array}$ \\
\hline Charpentier, entretien & $\begin{array}{l}\text { Syndiqué } \\
\text { Non syndiqué }\end{array}$ & $\begin{array}{l}128,1 \\
135,1\end{array}$ & $\begin{array}{l}123,8 \\
132,7\end{array}$ & $\begin{array}{l}124,6 \\
131,9\end{array}$ & $\begin{array}{l}123,0 \\
134,6\end{array}$ & $\begin{array}{l}124,3 \\
130,0\end{array}$ & $\begin{array}{l}121,2 \\
130,0\end{array}$ \\
\hline Tuyauteur, entretien & $\begin{array}{l}\text { Syndiqué } \\
\text { Non syndiqué }\end{array}$ & $\begin{array}{l}144,9 \\
162,2\end{array}$ & $\begin{array}{l}134,3 \\
166,9\end{array}$ & $\begin{array}{l}133,7 \\
159,7\end{array}$ & $\begin{array}{l}132,0 \\
162,2\end{array}$ & $\begin{array}{l}135,9 \\
164,1\end{array}$ & $\begin{array}{l}132,5 \\
163,1\end{array}$ \\
\hline Plombier, entretien & $\begin{array}{l}\text { Syndiqué } \\
\text { Non syndiqué }\end{array}$ & $\begin{array}{l}128,9 \\
146,0\end{array}$ & $\begin{array}{l}128,4 \\
143,0\end{array}$ & $\begin{array}{l}126,4 \\
145,6\end{array}$ & $\begin{array}{l}123,3 \\
148,3\end{array}$ & $\begin{array}{l}127,0 \\
144,5\end{array}$ & $\begin{array}{l}125,4 \\
144,5\end{array}$ \\
\hline $\begin{array}{l}\text { Mécanicien de machines fixes, } \\
\text { première classe }\end{array}$ & $\begin{array}{l}\text { Syndiqué } \\
\text { Non syndiqué }\end{array}$ & $\begin{array}{l}147,3 \\
180,2\end{array}$ & $\begin{array}{l}142,9 \\
180,0\end{array}$ & $\begin{array}{l}138,6 \\
181,0\end{array}$ & $\begin{array}{l}135,0 \\
192,2\end{array}$ & $\begin{array}{l}128,9 \\
173,9\end{array}$ & $\begin{array}{l}130,4 \\
180,5\end{array}$ \\
\hline $\begin{array}{l}\text { Journalier, non-production } \\
\text { (taux de rémunération horaire moyen) }\end{array}$ & $\begin{array}{l}\text { Syndiqué } \\
\text { Non syndiqué }\end{array}$ & $\begin{array}{l}4,03 \$ \\
3,41 \$\end{array}$ & $\begin{array}{l}4,80 \$ \\
4,00 \$\end{array}$ & $\begin{array}{l}5,50 \$ \\
4,43 \$\end{array}$ & $\begin{array}{l}6,11 \$ \\
4,76 \$\end{array}$ & $\begin{array}{l}6,37 \$ \\
5,10 \$\end{array}$ & $\begin{array}{l}7,03 \$ \\
5,51 \$\end{array}$ \\
\hline
\end{tabular}

${ }^{1}$ Le taux de rémunération horaire moyen de chaque catégorie professionnelle spécialisée est exprimé en pourcentage du taux de la catégorie non spécialisée (journalier). Un taux de $100 \%$ signifierait qu'il y a égalité de rémunération entre une catégorie spécialisée et la catégorie non spécialisée.

${ }^{2}$ Le statut de syndiqué (ou de non syndiqué) signifie que plus (ou moins) de $50 \%$ des travailleurs d'une catégorie donnée dans une entreprise sont représentés par une unité de négociation.

Source: Données du ministère du Travail. 
qu'ils doivent leur verser. En abaissant le coût des salaires, la création directe d'emplois peut donc corriger les distorsions de ce genre ${ }^{23}$.

Officiellement destinés à réduire le chômage, les programmes de création d'emplois viseraient donc à contrer «le rétrécissement des écarts [salariaux qui] peut être imputable à la progression plus rapide des salaires de base que des rémunérations offertes aux échelons supérieurs » ${ }^{24}$ et, conséquemment, à rectifier «le processus d'allocation des travailleurs»! On doit ainsi comprendre que ce processus, relié par le C.E.C. à la possibilité d'un large redéploiement des emplois, devrait mieux traduire une adaptation de l'évolution de la division internationale du travail aux changements technologiques jugés inévitables et urgents pour conserver ou améliorer ses avantages concurrentiels au sein de l'économie mondiale.

Ce «redéploiement des emplois» dont parle le C.E.C. suggère en fin de compte une adhésion au principe de la mobilité de la main-d'œuvre. Mobilité géographique, d'abord, qui se trouverait, selon John Hayes, grandement entravée par les arrangements constitutionnels en vigueur au Canada, à un degré même plus poussé, sous certains aspects, qu'au sein de la Communauté économique européenne (C.E.E.) ${ }^{26}$. Mais aussi et peut-être surtout, mobilité sectorielle qui se grefferait à un programme visant à «adapter l'industrie canadienne aux nouvelles réalités de la concurrence internationale» et comportant, "entre autres, l'adaptation des techniques, la réorganisation des usines

23. Ibid., 115-116. Le «budget de relance» présenté le 19 avril 1983 par le gouvernement canadien peut au moins donner prise à des interprétations pas nécessairement orthodoxes!

24. Ibid., 75 .

25. Ibid., 2.

26. John A. Hayes, Economic Mobility in Canada. A Comparative Study, Étude préparée pour le ministère de l'Industrie et du Commerce et le Bureau des relations fédérales-provinciales, Gouvernement du Canada, Approvisionnements et Services Canada, Ottawa, 1982, 52. 
et des méthodes de production et, dans certains cas, l'abandon complet des opérations, avec tout ce que cela comporte du point de vue du redéploiement des ressources concernées (les travailleurs, notamment) vers d'autres activités » ${ }^{27}$. Ce programme, ce n'est sûrement pas le Président de la Banque de Montréal qui s'en dissocie quand il pose que, pour le Québec, "la solution, c'est de remplacer des industries par d'autres, d'en créer de nouvelles, dans des secteurs en pleine croissance, générateurs d'emplois. Mais on ne peut sortir quelqu'un de sa filature et le mettre le lendemain à fabriquer des circuits intégrés; il y a tout un processus d'adaptation entre les deux... ${ }^{28}$ Et s'il avait poursuivi dans l'esprit de plusieurs énoncés du C.E.C., il aurait sans doute désigné les «inutiles rigidités» introduites par les conventions collectives négociées avec les syndicats!

En somme, si l'on s'est rarement aventuré à réclamer la disparition des syndicats, il apparaît bien clair que ceux-ci peuvent difficilement esquiver l'attaque. Du moins sont-ils contraints de se renier en bonne partie s'ils acceptent que les sources des problèmes économiques se ramènent fondamentalement à des taux de salaires trop élevés, à des taux de rémunération globale trop coûteux, à des écarts salariaux insuffisants, à une mobilité géographique et sectorielle trop limitée de la main-d'œuvre, etc. C'est pourtant autour de ces enjeux que s'articule l'offensive anti-syndicale qu'il est maintenant possible d'observer à travers diverses interventions patronales et gouvernementales destinées à réduire la résistance à une réorganisation du travail. D'autant plus que les gains des syndicats comportent couramment des effets d'entraînement au-delà même des rangs syndicaux.

27. Conseil économique du Canada, Pour un commun avenir. Une étude des relations entre le Canada et les pays en développement, Approvisionnements et Services Canada, Hull, 1978, 59.

28. William D. Mulholland, «Le monde vu par un banquier», entrevue publiée dans L'actualité, vol. 8, no. 4, avril 1983, 14. 
Il semble juste d'observer qu'au Canada, la sécurité d'emploi, la sécurité au travail, les procédures de griefs et les dispositions connexes résultent essentiellement du processus de la négociation collective. On ne trouve donc ces protections, de façon formelle, que dans les secteurs syndicalisés; les gouvernements de toutes les provinces ont [cependant] dû fournir une protection minimale correspondante pour les secteurs non syndicalisés, par des lois sur les relations de travail. ${ }^{29}$

La réponse syndicale à diverses invitations à la collaboration se fit sans doute trop attendre au goût de plusieurs.

\section{La réponse syndicale}

Dans l'analyse de la conjoncture actuelle, on peut toujours rappeler que «s'il avait fallu attendre la collaboration de la législation bourgeoise, les syndicats n'auraient jamais réussi à faire la percée qu'ils ont faite dans l'histoire du $19^{\mathrm{e}}$ et du $20^{\mathrm{e}}$ siècles, au Québec comme ailleurs dans le monde». Ainsi, si on doit reconnaître "que l'attaque patronale contre les syndicats atteint un rare degré de brutalité dans la présente conjoncture de crise économique», on se hâte de souligner que "la tiposte des travailleurs demeure néanmoins possible et nécessaire ${ }^{30}$. Cependant, il faut bien souligner que la «riposte» syndicale a perdu beaucoup de son intensité au cours des plus récentes années. Les réclamations du mouvement ouvrier n'ont pas radicalement changé au cours des dix dernières années; il faut toutefois noter que la force politique du mouvement syndical s'est trouvée grandement amoindrie ces derniers temps. La collaboration, carrément rejetée il y a seulement quelques années, apparaît ou semble du moins apparaître beaucoup moins repréhensible.

29. Conseil économique du Canada, Des travailleurs et des emplois. Une étude du marché du travail au Canada, Résumé et recommandations, 1976, 56.

30. Marielle Désy, Marc Ferland, Benoît Lévesque et Yves Vaillancourt, La conjoncture au Québec au début des années 80: les enjeux pour le mouvement outrier et populaire, La Librairie socialiste de l'Est du Québec, Rimouski, 1980, 137. 
Ainsi, seulement quelques mois, on pourrait même parler de quelques semaines, après l'adoption de la loi 111 dont on a assez souvent fait ressortir la sévérité exemplaire et sans précédent et après celle des lois 68,70 et 105 qui réduisaient quasiment à rien le pouvoir de négociation des employés des secteurs public et parapublic, les dirigeants de trois centrales, la Fédération des travailleurs du Québec (F.T.Q.), la Confédération des syndicats nationaux (C.S.N.) et la Centrale des syndicats démocratiques (C.S.D.), acceptaient de participer, le 30 mars 1983, à une rencontre à huis clos convoquée par le Premier ministre québécois pour discuter de relance économique. «Fait à signaler, cette rencontre ne portait que sur les problèmes du secteur privé et on a volontairement escamoté le volet des négociations dans le secteur public. ${ }^{31}$ "Un témoin privilégié des démarches qui avaient préparé cette rencontre soutenait, seulement quelques jours avant sa tenue, que les pressions en faveur de ces exercices de concertation provenaient avec une insistance particulière du monde syndical. Son témoignage était en grande partie confirmé, deux semaines plus tard, par une intervention «spectaculaire» du Président de la C.S.N. Celui-ci, lors d'une réunion du Conseil confédéral de la centrale tenue à la mi-avril, invitait ceux qui s'opposaient à toute forme de concertation à s'élire de nouveaux dirigeants s'ils le jugeaient approprié.

Si le problème réel est un problème de confiance, réglons-le comme tel. Mais ne nous empêchons pas par la méfiance systématique, par les rumeurs sourdes de corridors, par les jeux purement politiques, d'aller en tout état de cause et dans toutes les circonstances défendre partout les travailleurs qui nous confient le mandat de les représenter. ${ }^{32}$

Manifestement, on se trouve maintenant assez éloigné de la situation qui prévalait au début des années soixante-dix, du

31. Voir «Lévesque a présidé un 'mini-sommet' en secret», dans Le Devoir, 6 avril 1983,3 .

32. Voir Pierre Pelchat, "Donatien Corriveau déplore l'absence de concertation», dans Le Soleil, 16 avril 1983, A4. 
moins selon la lecture qui en était alors faite à travers le Rapport Fantus.

The extremist positions adopted by provincial union leadership and their potential effect on the attitudes of the rank and file will be a continuing cause of concern. Industrial investors will also be worried about the possibility of a spillover into the private sector of the labor relations problems which have arisen in the public sector ${ }^{33}$.

À la suite de la rencontre du 30 mars dont il fut question ci-haut, le Président du C.P.Q. soulignait le «vrai désir de dialogue» qui animait les participants et parlait de réunion des "gens de la famille»!

Pour les tenants des explications des comportements sociaux articulées autour de l'existence d'élites ou d'oligarchies, le changement de leadership syndical constituerait sans doute la source la plus importante de cette réorientation des attitudes syndicales. Il importe tout de même de rappeler que la mobilisation, potentielle et souvent réelle, d'une bonne partie du mouvement ouvrier représentait il y a seulement dix ans une «menace» beaucoup plus grande que ce n'est présentement le cas. Ainsi, en 1973, il avait suffi que la C.S.N. entreprenne une tournée visant à «informer les syndicats de la teneur et des conséquences du bill $89{ }^{34}$ pour que le gouvernement Bourassa décide de ne pas aller au-delà de la première lecture de ce projet de loi. Ce dernier, intitulé Loi assurant le bien-être de la population en cas de confit de travail, avait pour objectif de réglementer le droit de grève dans les services publics et d'assurer, en pareilles circonstances, le maintien des services essentiels. On prétendait qu'en étant adopté, le projet de loi 89 deviendrait " un boomerang» et qu'ainsi «ceux qui se [réjouissaient] d'enfin mater les

33. The Fantus Company (Chicago), Industrial Development in Queber, Etude préparée pour le ministère de l'Industrie et du Commerce, Gouvernernent du Québec, Québec, mai 1972, III, 10.

34. Confédération des syndicats nationaux, 89. Le bill Fantus, 1973, p. 11. 
syndicats, pour exploiter plus à leur aise les travailleurs, [connaîtraient] les jours sombres qu'ils auront eux-mêmes provoqués ${ }^{35}$. Le discours syndical avait alors une grande crédibilité. Car on avait nettement le sentiment qu'il ne s'agissait pas de simples discours. La grogne syndicale, on le savait, n'était pas le fait des seuls dirigeants.

Il se révèle pertinent de signaler qu'en l'espace de quelques années, la position des organisations syndicales comme organisations représentatives de l'ensemble des travailleurs a eu fortement tendance à se détériorer. Cette situation contraste singulièrement avec ce que l'on avait pu observer au cours des années soixante: «de 1961 à 1967, le nombre de syndiqués augmente de $7 \%$ par rapport à la main-d'ouvre active. Cette croissance est surtout due aux remarquables progrès que fait le syndicalisme dans deux secteurs d'activités jusque-là marginalement organisés: le commerce et, surtout, le secteur public et parapublic ${ }^{36}$. Comme le souligne Jean-Marc Piotte, «la valorisation de la fonction publique et para-publique impliquait [...] qu'on accorde à ces employés les moyens syndicaux pour qu'ils conquièrent cette 'valorisation' ${ }^{37}$. Si on se souvient qu'en 1974 , les syndicats représentaient $34.2 \%$ de la population active et $41.3 \%$ des travailleurs rémunérés au Québec, les chiffres reproduits au tableau $4^{38}$ fournissent une bonne indication du déclin relatif du syndicalisme québécois. À l'évidence, la progression du chômage accentue les effets liés à l'absence de progression des effectifs.

35. Ibid., 3.

36. Jean-Marc Piotte, "Le syndicalisme au Québec depuis 1960 ", dans Diane Ethier, Jean-Marc Piotte et Jean Reynolds, Les travailleurs contre l'État bourgeois, avril et mai 1972, Les Éditions de l'Aurore, Montréal, 1975, 20.

37. Ibid., 31.

38. Tableau tiré de Secrétariat permanent des conférences socio-économiques du Québec, L'état de la situation socio-économique, La conférence au sommet, Québec, 1982. 59. 
TABLEAU 4

Taux globaux de syndicalisation, Québec, 1977-1979

\begin{tabular}{lcccc}
\hline Année & $\begin{array}{c}\text { Effectifs } \\
\text { syndicaux }\end{array}$ & $\begin{array}{c}\text { En \% de la } \\
\text { population active }\end{array}$ & $\begin{array}{c}\text { En \% de la main- } \\
\text { d'cuvre potentielle }^{\text {En }}\end{array}$ & $\begin{array}{c}\text { En des travailleurs } \\
\text { rémunérés }\end{array}$ \\
\hline $1977^{\mathrm{a}}$ & 850750 & 30,5 & 32,9 & 37,0 \\
1978 & $815882^{\mathrm{b}}$ & $28,9^{\mathrm{c}}$ & $32,4^{\mathrm{c}}$ & $36,9^{\mathrm{c}}$ \\
1979 & $849318^{\mathrm{b}}$ & $29,5^{\mathrm{d}}$ & $32,5^{\mathrm{d}}$ & $36,4^{\mathrm{d}}$
\end{tabular}

a Les taux pour l'année 1977 sont tirés de F. Delorme. « Quelques données sur la syndicalisation au Québec en 1977 " dans la revue Le marché du travail, volume 1, numéro 1, 1979, pp. 34 à 38. CRSMT, ministère du Travail de la Main-d'ceuvre et de la Sécurité du revenu.

b Les données sur les effectifs syndicaux proviennent de Loi sur les déclarations des corporations et des syndicats ouvriers, rapport 1979, partie 11, syndicats ouvriers. Statistique Canada. 71-202

c Moyennes annuelles de la population active, Statistique Canada. 7 I-529

${ }^{d}$ La population active, Statistique Canada. 7 1-001

e On exclut de la population active les travailleurs indépendants ainsi que les travailleurs familiaux non rémunérés

f Le concept de travailleurs rémunérés exclut aussi bien les chômeurs que les travailleurs indépendants ainsi que les travailleurs familiaux non rémunérés

Un des effets immédiats de cette tendance est de miner la prétention des centrales syndicales à leur «responsabilité conjointe quant à la défense des intérêts de l'ensemble des travailleurs du Québec» ${ }^{39}$. Cette prétention constitue l'objet de nombreuses attaques, d'autant plus que l'on insiste régulièrement pour faire ressortir que les taux globaux de syndicalisation tendent à masquer le fait que seulement un peu plus d'un travailleur sur cinq est syndiqué dans le secteur privé comparativement à près de trois sur cinq dans ce que l'on définit comme le secteur public ${ }^{40}$. Telle concentration des effectifs syndicaux

39. Centrale de l'enseignement du Québec, Mémoire de la Centrale de l'enseignement du Québec sur le projet de loi no. 126 portant sur les conditions minimales de travail, mars 1979, 1.

40. Ces données, valables pour 1976, sont tirées de Secrétariat permanent des conférences socio-économiques du Québec, L'état de la situation socio-économique, op. cit., 60. On y précise: «Le secteur public comprend les travailleurs ceuvrant au sein de l'administration publique ainsi que dans l'enseignement et les services médicaux et sociaux. Le secteur privé se définit par complément au secteur public.» 
explique vraisemblablement pourquoi, de ces temps-ci, l'offensive contre les syndicats apparaît viser spécialement ceux qui représentent les employés du secteur public. D'ailleurs, les porte-parole de ces syndicats doivent bien être conscients que les effets d'entrainement qu'ils veulent rattacher aux gains obtenus par leurs affiliés du secteur public peuvent aussi jouer en sens inverse. Le C.E.C., pour sa part, l'assume assez explicitement.

Aux États-Unis, les lignes directrices obligatoires à l'égard des salaires dans le secteur public ont eu des répercussions importantes sur les hausses de traitement des employés fédéraux. Le plafond de 4,8\% imposé par l'administration Reagan a probablement contribué à ralentir la progression des salaires dans ce pays ${ }^{4}$.

Les organisations syndicales réalisent évidemment que «dans sa stratégie de négociation avec le secteur public, le gouvernement se sert amplement de cette sous-syndicalisation des travailleurs du secteur privé», particulièrement quand il établit ses comparaisons «entre les deux secteurs ${ }^{42}$. La réponse proposée par les syndicats consiste plutôt à affirmer que «l'accès massif à la syndicalisation est la seule façon d'ouvrir la voie à de vrais changements au Québec ${ }^{43}$. On a bien tenté, de plus, de montrer que tous les syndiqués étaient confrontés à «un même problème de fond: les retombées d'une crise économique dont les travailleurs et les travailleuses écopent le plus durement, tant dans le secteur privé que dans le secteur public ${ }^{44}$. Mais, du côté patronal et gouvernemental, l'heure n'est pas aux expressions de sympathie sur le sort réservé aux syndiqués dans la recherche de solutions à la crise.

41. Conseil économique du Canada, Les temps difficiles, op. cit., 107.

42. "La crise, ça se combat!", dans Le monde outrier, mars 1982, 13.

43. "Seul l'accès massif à la syndicalisation ouvrira la porte à un vrai changement ", dans Le monde outrier, juin 1980, 8.

44. «Coupures et fermetures: une même lutte», dans Le monde outrier, avril $1982,7$. 
Lors de la présentation par la C.E.Q. et la C.S.N. d'un mémoire conjoint au nouveau gouvernement québécois, le Premier ministre et quelques-uns de ses Ministres s'étaient appliqués à faire l'éloge du mouvement syndical et à le rassurer. Pendant qu'un Ministre qualifiait de «réalistes» les revendications présentées sur les lois du travail, les services publics, les mesures économiques et sociales, etc., un autre ne ménageait pas ses termes: «Ce mémoire est complet. Il arrive à point nommé et je voudrais vous rassurer sur le fait que le développement économique du Québec ne se fera pas sur le dos des travailleurs ni du point de vue santé ni du point de vue économique. » Le Premier ministre lui-même avait l'évaluation généreuse: «vous venez de faire le plus impressionnant des tours d'horizon, la plus impressionnante des analyses, qui nous ait été présentée depuis notre arrivée au pouvoir ${ }^{45}$. Six ans plus tard, lors de la présentation de son discours inaugural du 23 mars 1983, le Premier ministre se montrait beaucoup plus enclin à jouer le rôle du préfet de discipline. Laissant grandement tomber son penchant généreux, il s'attardait à évoquer la nécessité de «changer comme société». Selon ses propos, l'État devait «manifester une plus grande faculté d'adaptation aux défis nouveaux et introduire plus de souplesse dans son fonctionnement, notamment en responsabilisant davantage ses employés, à quelque niveau que ce soit ${ }^{46}$.

Son message sur le sens des responsabilités s'adressait officiellement à tous mais visait plus spécialement, comme le texte le montre abondamment, les syndiqués des services publics. Ce message se greffait par ailleurs assez bien aux exhor-

45. Réactions reproduites dans Centrale de l'enseignement du Québec et Confédération des syndicats nationaux, Mémoire commun de revendications présenté par la C.S.N. et la C.E.Q. au gouvernement du Québec, 28 février 1977, 62.

46. René Lévesque, Discours inaugural, 23 mars 1983, Extraits reproduirs dans Le Soleil, 24 mars 1983, A-13. 
tations nombreuses formulées à l'endroit de l'ensemble du monde syndical et traduites explicitement par Gérard Dion.

[...] il va falloir que les syndicats eux-mêmes s'ajustent au contexte économique, social et politique de notre époque et tiennent compte de la conjoncture dans laquelle nous nous trouvons. Le monde a évolué depuis cent ans: l'économie, les techniques de production, le marché des produits, la condition des travailleurs ne sont plus les mêmes.

Depuis une trentaine d'années toutes les institutions se sont questionnées sur leur nature, leur rôle, leur stratégie d'action pour s'ajuster. On a questionné l'État, les entreprises de l'intérieur et de l'extérieur; même l'Église catholique l'a fait. [...] Pourquoi le syndicalisme serait-il la seule institution à refuser de faire son auto-critique et à traiter d'antisyndical quiconque ose aborder un pareil problème? ${ }^{47}$

La réponse à cette dernière question, les travailleurs l'ont amplement exprimée, depuis déjà quelque temps, avec la conviction qu'ils continuaient à payer la note malgré les tentatives de s' «ajuster au système économique. Plus on se débat, plus on cale ${ }^{48}$. Le fort scepticisme qui se trouve ainsi traduit est en pratique relié autant aux changements technologiques qu'aux nombreuses fermetures d'entreprises et aux multiples licenciements collectifs qui en découlent.

Ces changements [technologiques], particulièrement importants en cette période de crise, où le capital tente par tous les moyens de rentabiliser ses investissements, se font le plus souvent au détriment de leurs conditions de travail et de vie. Une étude récente, réalisée au Canada auprès de 102 firmes industrielles et commerciales, démontre que pour chaque nouveau poste créé suite à des changements technologiques, au moins trois employé(e)s sont mis à pied. Cela sans parler du risque d'une déqualification et d'un contrôle plus accentué du travail que teprésentent de tels changements pour la majorité des travailleurs et des travailleuses, sans parler également de nouveaux dangers qu'ils devront affronter pour leur santé ${ }^{49}$.

47. Gérard Dion, «D'abord enrayer la désyndicalisation» dans Le Devoir, 20 avril 1983,7 .

48. Confédération des syndicats nationaux, $l l n^{\prime} y$ a plus d'avenir pour le Québec dans le système économique actuel. La C.S.N. propose un changement radical, 1971, 3.

49. Confédération des syndicats nationaux, Du travail pour tout le monde, Manifeste des travailleurs et travailleuses victimes de fermetures d'entreprises et de licenciements collectifs, 1982, 19. 
Si chacun prenait finalement son mal en patience et acceptait de se sacrifier pour l' « intérêt général ", on s'adonnerait sans doute à exprimer sympathies et sentiments de "solidarité morale» pour admettre les effets coûteux, mais nécessaires pour réussir la restructuration de l'économie imposée par les «dures réalités» de la concurrence internationale. C'est d'ailleurs en puisant à cette réalité que l'on justifie régulièrement les nécessaires compressions du secteur étatique. Cependant, à partir du moment où des organisations de travailleurs osent dire que ces conditions font que «les affrontements avec la classe ouvrière vont s'intensifier ${ }^{50}$, la sympathie semble rapidement laisser place à l'agacement. Cet agacement se trouve aiguisé si l'on ajoute que «tous doivent prendre conscience que les enjeux dépassent maintenant leur quotidienneté et nous projettent dans la spirale de la mondialisation électronique et des «tiques» accrochés au cul des nouvelles théories" ${ }^{51}$. À une époque où l'on s'évertue à démontrer l'urgence de prendre le virage technologique, la réplique est grandement prévisible. Elle vient d'un $\mathrm{Mi}$ nistre du gouvernement Lévesque.

Il y a encore des militants engagés dans le Parti québécois qui croient dans le Québec, mais qui sont encore victimes de ces égoïsmes de classes. Je ne peux comprendre qu'on soit à la fois dévoué à l'idée d'un Québec et en même temps incapable de faire des arbitrages quand son intérêt personnel est en cause. Il va falloir que notre société mûrisse. Elle va mûrir uniquement si on lui montre le chemin du dépassement ${ }^{52}$.

50. Centrale de l'enseignement du Québec, Négociation C.E.Q. Animation, (Pour des solutions sociales et progressistes à la crise), février 1982, 21.

51. Jean-Claude Tardif, «Quand le travail s'ébarouit, le travailleur prend le rhume», dans Magazine C.E.Q., (Économie), Hiver 1982, 11.

52. Ptopos du ministre Yves Bérubé cités dans Centrale de l'enseignement du Québec, Le droit de grève: outil de la démocratie, facteur de progrès social, Mémoire présenté à la Commission parlementaire du travail et de la main-d'ouvre sur les moyens d'améliorer les négociations et l'exercice du droit de grève, septembre 1981, 22. 


\section{Des appels à un effort national et «corporatiste »}

Le «dépassement» exigé par les dirigeants québécois suggère un certain retour à l'époque du capitalisme naissant quand l'on s'efforçait de mettre «en contraste les conséquences heureuses des activités dictées par l'intérêt avec les calamités que déchaîne le libre jeu des passions ${ }^{53}$. Traduit dans le langage d'un autre Ministre québécois, cela voulait plus spécialement dire qu'il fallait mettre fin à toute relation conflictuelle, donc passionnée: «Il importe que tous les agents économiques s'efforcent d'éliminer les conflits inutiles et fournissent une contribution tangible à l'accroissement de la productivité industrielle et commerciale, que tous se sentent responsables de l'état de l'économie québécoise ${ }^{54}$.

Bien sûr, malgré une volonté ferme de "structuration du dialogue du Québec», on devait bien reconnaître la quasiimpossibilité de «faire disparaître les intérêts particuliers, même opposés, des groupes qui composent la société ${ }^{55}$. L'émergence d'un esprit de concertation au sein d'une société «trop fragmentée» demeurait tout de même un objectif qui devait permettre de «déboucher sur certaines formes de consensus. D'où la nécessité de [...] se parler avec respect et sans se prêter d'intentions belliqueuses dès le départ » ${ }^{56}$. Le discours du gouvernement québécois sur la nécessité de la concertation puisait par ailleurs à des sources bien « respectables».

53. Albert O. Hirschman, Les passions et les intérêts. Justifications politiques du capitalisme avant son apogée, Coll. Sociologies, Presses universitaires de France, Paris, 1980 (1977), 34.

54. Propos de l'ex-ministre Rodrigue Tremblay, cités dans Centrale de l'enseignement du Québec, Le développement économique au service du peuple québécois, Point de vue sur la réalité socio-économique du Québec, Sommet économique de Pointe-au-Pic, 24 26 mai $1977,9$.

55. Secrétariat permanent des conférences socio-économiques, Le bilan des conférences socio-économiques du Québec, de mai 1977 à décembre 1978, mars 1979, 93.

56. Discours d'ouverture du Premier ministre, dans Rapport, Le sommet économique, Pointe-au-Pic, mai 1977, 14. 
Pour progresser, le Québec doit être compétitif, non seulement avec les autres parties du Canada, mais aussi avec le reste du monde pour ce qui est des biens et services qu'il produit. Nous estimons que l'État, les patrons, les syndicats ouvriers et les travailleurs ont des responsabilités collectives et partagées en ce domaine ${ }^{57}$.

Le gouvernement péquiste ne fit en fin de compte qu'ajouter l'ingrédient de la conscience nationale: «L'émergence d'une conscience nationale et la volonté de concertation des agents économiques sont indispensables à une articulation des moyens à mettre en ouvre pour assurer le développement du Québec tant au point de vue économique que social et culturel $\gg 58$. On comprend ainsi mieux ce que cela veut dire quand on proclame que «la souveraineté, c'est d'abord et avant tout un état d'esprit, une manière de vivre et une manière de fonctionner ${ }^{59}$ ! Du côté syndical, on s'est cependant montré réticent à participer à la construction de solidarités nationales élaborées «pour unifier le tout, pour cimenter [des] agents économiques aux intérêts 'divergents' $\gg 60$.

L'insistance du gouvernement québécois à affirmer l'insuffisance «de conscience nationale et de concertation des agents économiques indispensables à une articulation des moyens à mettre en ouvre pour assurer le développement économique, social et culturel ${ }^{61}$ a incité bon nombre d'observateurs à percevoir une orientation corporatiste à travers ces appels. Pour me-

57. Chambre de commerce de la province de Québec, Politiques d'action/75, Montréal, 1975, 38.

58. Gouvernement du Québec (Ministère d'État au Développement économique), Bâtir le Québec. Énoncé de politique économique, Éditeur officiel du Québec, Québec, $1979,100$.

59. Énoncé retracé dans un feuillet conçu par le Parti québécois pour sa campagne de financement du printemps 1983.

60. Centrale de l'enseignement du Québec, BATTIR LE QUÉBEC ou l'exploitation tranquille de la majorité, Édition spéciale de la revue Ligne directe, février 1980, 11.

61. Gouvernement du Québec (Ministère d'État au Développement économique), Bâtir le Québec, op. cit., 61. 
surer la justesse de cette perception, il convient tout d'abord de proposer une définition du corporatisme, empruntée à Léo Panitch: «une structure politique par laquelle, au sein du capitalisme avancé, les groupes socio-économiques organisés et rattachés à la production se trouvent intégrés au moyen d'un système de représentation et de rapports de coopération entre leaders et d'un système de mobilisation et de contrôle social en ce qui touche les masses ${ }^{62}$. Pierre Elliott Trudeau a voulu voir, dans le passé, «la preuve la plus accablante de notre monolithisme idéologique» à travers «l'univocité, la constance, l'enthousiasme» qui avaient entouré la propagation de «l'évangile corporatiste ${ }^{63}$. Ceci ne justifie pas cependant de se représenter le corporatisme par sa seule formulation idéologique ${ }^{64}$. En ce sens, les orientations apparemment corporatistes qui sous-tendent les discours sur la collaboration responsable et raisonnable ne devraient duper personne sur la portée effective de ces discours.

Un conditionnement idéologique se révèle évidemment préalable à l'établissement du corporatisme, mais il ne le constitue pas. Ce que les responsables politiques traduisent bien en rappelant qu' «il ne suffit pas, bien sûr, de lancer un appel à la solidarité pour que la concertation se réalise. La concertation et la coordination dans l'action nécessitent en effet un long cheminement, partant d'une même perception de la réalité et de la nécessité de travailler en commun. " Ce long cheminement apparaît d'ailleurs essentiellement requis pour permettre « d'amor-

62. Léo Panitch, "Corporatism in Canada?", dans Richard Schultz, Orest M. Kruhlak et John C. Terry (eds), The Canadian Political Process, Holt, Rinehart and Winston of Canada, Toronto, 3ème éd., 1979 (1979), 53.

63. Pierre Elliott Trudeau, «La province de Québec au moment de la grève», dans Pierre Elliort Trudeau (ed.), La grève de l'amiante, Les Éditions du Jour, Montréal, $1970,35$.

64. Léo Panitch, «Corporatism in Canada?», op. cit., 53. 
cer un dialogue constructif qui devra inévitablement conduire à des concessions mutuelles $\gg 65$.

Des organisations syndicales ont exigé une participation pleine et égalitaire de manière à éviter que les concessions exigées ne visent que les salaires et revenus d'emploi tout en négligeant de demander des comptes aux détenteurs du capital ${ }^{66}$. D'autres ne voulaient surtout pas contribuer à «créer l'impression que les travailleurs, en particulier les syndiqués, sont l'un des facteurs de cette crise [...], alors qu'ils en sont d'abord les cibles et les victimes, tout comme le sont les classes populaires en général " ${ }^{67}$. Les réticences syndicales trouvaient d'ailleurs confirmation à travers les tentatives d'implantation de la concertation. Ainsi, les représentants du monde patronal et de l'entreprise ont clairement manifesté leur opposition à un partage égal $\mathrm{du}$ pouvoir entre les divers agents: le capital, le travail et l'État ${ }^{68}$.

Les résultats des initiatives de l'État favorisant la concertation se révélèrent en réalité variés. Du côté d'Ottawa, on semble avoir mieux réussi, au moins plus rapidement; en parvenant à réunir en juillet 1978 « cinq représentants du monde industriel, cinq membres du bureau du Congrès du travail du Canada, un professeur d'université et un président choisi dans le secteur privé» au sein d'un comité chargé «de faire des recommandations $[\ldots]$ pour une politique générale de l'économie» ${ }^{69}$. Ce

65. Gouvernement du Québec (Ministère d'État au Développement économique), Bâtir le Québec, op. cit., 85.

66. Congrès du travail du Canada, «Labour's Manifesto for Canada», dans Robert K. Logan (ed.), The Way Abead for Canada. A Paperback Referendum, Lester and Orpen, Toronto, 1977, 98-99.

67. Centrale de l'enseignement du Québec, Propositions pour une sortie de crise à l'avantage de la majorité, Conférence au sommet de Québec, 5-7 avril 1982, 2.

68. Richard W. Phidd et G. Bruce Doern, The Politics and Management of Canadian Economic Policy, The Macmillan Company of Canada, Toronto, 1978, 548.

69. Conseil des ministres au Développement économique, Vers la croissance industrielle. Poursuite du dialogue, Gouvernement du Canada, Otrawa, 1979, 1. 
«comité de synthèse» poursuivait en pratique le travail entrepris auparavant par un ensemble de 23 groupes de travail sectoriels mis sur pied à la suite de la conférence fédérale-provinciale des Premiers ministres sur l'économie qui s'était tenue en février $1978^{70}$. Le gouvernement du Québec a peut-être mis plus de temps à atteindre son objectif de concertation, mais les expériences les plus récentes de rencontres au sommet dont il fut question plus haut tendent à faire croire qu'il y a eu un rattrapage significatif.

Les éléments présentés dans les premières sections de cet exposé font cependant ressortir que le rapprochement constaté ne découle pas de gains favorisant les organisations de travailleurs! D'ailleurs, les multiples arguments avancés pour montrer la nécessité des compressions salariales permettent difficilement d'envisager des orientations différentes. Il reste effectivement peu de place pour le doute quant aux effets sur les syndicats d'une politique de restructuration des économies «nationales» visant à ajuster et adapter ces dernières aux transformations profondes de l'économie mondiale. Dans l'énoncé de sa politique de troisième option, en 1972, le gouvernement canadien jugea approprié de rappeler que «la coopération étroite du gouvernement, du monde des affaires et du travail tout au long des étapes de l'application de cette politique serait essentielle» et il estima devoir «aussi s'employer à créer un climat propice à l'expansion des entreprises canadiennes ${ }^{71}$. Il était cependant révélateur, et grandement révélateur, que l'on précise dès ce moment ne pas prévoir "que cette option doive entraîner des

70. Douglas Brown et Julia Eastman, The Limits of Consultation. A Debate Among Ottawia, the Provinces and the Private Sector on an Industrial Strategy, Industrial Policy Series, Conseil des sciences du Canada/Institute of Intergovernmental Relations (Queen's University), Approvisionnements et Services Canada, Ottawa, 1981, 42.

71. Mitchell Sharp, «Relations canado-américaines: choix pour l'avenir», dans Perspectives internationales, numéro spécial, automne 1972, 20-21. 
modifications radicales dans les relations entre le gouvernement et le monde des affaires, même si le gouvernement devait alors suivre de plus près encore l'orientation de l'économie» ${ }^{72}$. En d'autres termes, les concessions n'avaient pas à venir prioritairement de ce côté...

De ce qui précède, on conçoit que tout effort d'implantation d'un fonctionnement corporatiste doit nécessairement se traduire par des relations tripartites et dépasser les rapports bipartites État-patronat ou État-syndicats ${ }^{73}$. Il ressort par ailleurs que l'ajustement des parties respectivement impliquées comporte, en cette phase plus spécifique de restructuration du capitalisme, plus d'exigences du côté syndical. Contrairement à ce qui a pu s'observer à l'époque de la crise des années trente, la phase actuelle de réorganisation de la production capitaliste conduit plus généralement à soutenir la nécessité d'une décroissance significative de l'intervention directe de l'État dans l'activité économique, pour le voir remplir avec plus de force ses rôles de planification et de gestion globale de l'emploi et des salaires ${ }^{74}$. Dans ces conditions, on en est graduellement venu à se convaincre que l'État « ne peut se permettre d'offrir des rémunérations qui dépassent toutes celles du secteur privé » 75 .

La politique de rémunération de l'État doit [...] tenter d'être le plus neutre possible quant aux prix et à l'emploi. Un écart salarial important entre les secteurs public et privé est source d'inflation et a des effets sur l'emploi. En effet, la rémunération du secteur public influence l'ensemble de la structure salariale et peut se traduire par un accroissement du chômage surtout dans une économie ouverte comme le Québec qui se doit de maintenir sa compétitivité ${ }^{76}$.

72. Ibid., 21.

73. Leo Panitch, «Corporatism in Canada?», op. cit., 54.

74. Idem.

75. Gouvernement du Québec (Ministère d'État au Développement économique), Bâtir le Québec, op. cit., 141.

76. Ibid., 140. 
C'est donc en tenant compte de ce contexte qu'il importe d'analyser la récente offensive contre les employés du secteur public et leurs organisations. Dans le but de faire partager les responsabilités de cette attaque, on a fait noter que «les travailleurs du secteur privé accepteront difficilement que leurs conditions de travail (salaires et sécurité d'emploi surtout) soient très inférieures à celles qui ont été déterminées dans le secteur public » ${ }^{77}$. L'aiguisement par les autorités politiques de ce ressentiment réel ne doit tout de même réussir à camoufler que les travailleurs du secteur privé se trouvent aussi visés, par association ou entraînement tout d'abord, mais aussi plus directement. En effet, même si l'on prend soin d'agrémenter ces propositions de la perspective d' "une plus grande participation des travailleurs à la vie de l'entreprise», les objectifs poursuivis laissent peu de place à l'ambiguïté: "Le nombre et la durée des grèves et des lock-outs, le taux d'absentéisme, la fréquence des accidents, les ralentissements de travail, les problèmes de productivité dénotent qu'il y a place pour de l'amélioration dans les relations patronales-ouvrières ${ }^{78} \mathrm{On}$ ne doit en somme pas perdre de vue que ces interventions se greffent à la politique de « redéploiement de l'économie qu'entend favoriser le gouvernement ${ }^{79}$ et que cette politique exige une main-d'œuvre responsable tant dans le secteur privé que dans le secteur public. Cela demeure vrai aussi bien du côté d'Ottawa que du côté de Québec. Et si jamais on y tient vraiment en haut lieu, on songera à un fonctionnement corporatiste dès lors que l'on aura réussi à

77. Ministère du Travail (Gouvernement du Québec), Le travail. Point de vue sur notre réalité, Le sommet économique du Québec, mai 1977, 17.

78. Ministère d'État au Développement économique, Le virage technologique. Bâtir le Québec - Phase 2. Programme d'action économique 1982-1986, Gouvernement du Québec, Québec, 1982, 211.

79. Gouvernement du Québec (Ministère d'État au Développement économique), Bâtir le Québec, op. cit., 272. 
«responsabiliser» à travers l'émergence d'une «conscience nationale »!

\section{L'effritement des solidarités syndicales}

Compte tenu de l'offensive qui se déployait avec force contre eux, les syndiqués du secteur public ont été amenés à se rendre compte que leurs « intérêts et [leurs] besoins coïncidaient avec ceux des autres travailleurs et travailleuses»; ils ont aussi tenté de croire que leurs solidarités s'étaient «élargies, sur le plan de la négociation, mais aussi sur [le plan...] politique» ${ }^{80}$. Cette assertion de la C.E.Q. ressemble cependant de plus en plus à un vœu qu'à une réalité solide. Il y a seulement quelques années, on était assez unanime à travers le mouvement syndical québécois à dénoncer "la politique des petits pas», ou de grignotage des droits syndicaux.

C'est une politique hypocrite et prenant les apparences de l'objectivité; c'est une politique d'autant plus redoutable et dont il faut absolument que nous prenions conscience. Devant cette politique, profondément vicieuse, il n'y a qu'une attitude possible: refuser absolument de céder du terrain. Les amendements proposé [au code du travail] sont mauvais en eux-mêmes, et ils sont mauvais au surplus parce qu'ils constituent une opération faisant partie, sans doute, d'une campagne à moyen et à long terme, qu'il est impérieux de contrer tout de suite ${ }^{81}$.

Au milieu des années soixante-dix, on partageait encore la conviction que toute attaque contre les syndicats des employés du secteur public représentait une attaque contre l'ensemble du mouvement syndical. Force est de constater un sérieux effritement de la solidarité syndicale. Et cela ne tient pas seulement à la rebuffade servie au Front commun des employés du secteur

80. Centrale de l'enseignement du Québec, Contre-réforme et appel au corporatisme. Propos sur un discours ministériel, Négociation C.E.Q. Animation, février 1982, 5.

81. Confédération des syndicats nationaux, Le gouvernement capitaliste contre te syndicalisme. Organisons la résistance, mai 1975, 6. 
public et parapublic lors du dernier affrontement avec l'État québécois en 1982-1983. Bien sûr, la C.E.Q. a constitué le dernier groupe à renoncer à la résistance contre l'attaque gouvernementale; mais ses membres furent régulièrement les derniers à le faire dans chaque Front commun précédent. Bien sûr, les syndiqués du secteur privé et ceux du secteur public sont devenus suspicieux les uns envers les autres et cela se répercute spécialement au sein de la C.S.N. où les fédérations du secteur privé se trouvent en position minoritaire ${ }^{82}$. En soulignant que «les fédérations du secteur privé commencent à relever la tête » à la C.S.N. ${ }^{83}$, il est tentant de suggérer, comme le fait le président de cette centrale, que «la stratégie de division du gouvernement entre les hauts et les bas salariés a fonctionné » ${ }^{84}$. Mais, déjà, au printemps 1976, on affichait peu de sympathie pour les professionnels syndiqués affiliés à la C.S.N. «en disant que «ces gens-là» ne croient pas vraiment au syndicalisme, "ils sont trop riches » " 85 !

En cherchant des explications à ce qui se passe à l'intérieur de la C.S.N., on pourrait être tenté de suggérer, comme on l'a fait pour interpréter la scission qui en 1972 a lourdement affecté cette centrale, que celle-ci n'a pas «su trouver l'heureuse alchimie qui lui aurait permis de conserver les anciens [les cols bleus des secteurs industriels en difficulté] et de faire une place aux nouveaux venus [les employés du secteur public et parapublic] ${ }^{86}$. Il faut cependant bien voir que le problème des rela-

82. Voir Pierre Pelchat, «La FAS au cœur du désaccord. Frictions entre élus du privé et du public», dans Le Soleil, 19 avril 1983, A2.

83. François Demers, «Syndicalisme: la grande dérive», dans le temps fou, no. 27, avril 1983, 29.

84. Pierre Pelchar, "Entrevue avec Donatien Corriveau. À l'heure des choix", dans Le Soleil, 23 avril 1983, B3.

85. Pierre Dupont et Gisèle Tremblay, Les syndicats en crise, Les grands dossiers de l'actualité, Les Éditions Quinze, Montréal, 1976, 53.

86. Jacques Rouillard, Histoire de la C.S.N. (1921-1981), Les Éditions du Boréal Express/Confédération des syndicats nationaux, Montréal, 1981, 242. 
tions entre syndiqués du secteur public et syndiqués du secteur privé déborde largement les problèmes de structure et de fonctionnement organisationnels. On peut bien proclamer que «la solidarité syndicale peut même dans l'avenir apporter une transformation profonde de la société ${ }^{87}$; on doit néanmoins admettre l'extrême fragilité et volatilité de cette solidarité actuellement. Tout au moins doit-on noter que la traduction organisationnelle de cette solidarité semble actuellement très mouvante.

Il est pertinent de constater que les réalignements du syndicalisme ont eu tendance à se manifester aux moments où il subissait les pires assauts du patronat et de l'État. Les attaques répétées du gouvernement Duplessis contre le mouvement syndical avaient sans doute contribué à un rapprochement des organisations syndicales dans les années cinquante, «des négociations [en vue d'une union organique s'étant] même engagées entre le C.T.C. [Congrès du travail du Canada] et la C.S.N. » ${ }^{88}$. Les rapports de plus en plus conflictuels entre l'État et ses employés expliquent aussi probablement en grande partie qu' «au début de 1977, la C.S.N. et la C.E.Q. [aient] mis sur pied conjointement un 'Comité de l'unité syndicale' [et aient] soumis un échéancier afin de permettre un large débat sur l'unité syndicale qui pourrait déboucher éventuellement sur la fusion organique des deux centrales " ${ }^{89}$. Il n'est vraisemblablement pas exagéré maintenant de soutenir que les syndiqués du secteur privé affiliés à la C.S.N. éprouvent plus de sympathie pour le «projet de Fonds de solidarité pour la création et le maintien de l'emploi» par lequel la F.T.Q. entend démontrer que «les travailleurs québécois sont prêts à s'impliquer activement pour favori-

87. Ibid., 307.

88. Paul Bélanger, «Mutations du syndicalisme québécois: hypothèse en vue d'une recherche", dans Fernand Harvey (ed.), Aspects bistoriques du mouvement ouvrier au Québec, Coll. Études d'histoire du Québec, Les Éditions du Boréal Express, Québec, $1973,194$.

89. (Collectif), 150 ans de luttes, op. cit., 205. 
ser une relance économique ${ }^{90}$ que pour toutes les dénonciations à l'emporte-pièce de l'autoritarisme et du «fascisme» du gouvernement Lévesque formulées par les syndiqués du secteur public.

En somme, la volonté des divers gouvernements de construire des solidarités nationales ne peut que forcer une remise en question des forces de l'action syndicale. Plus remarquablement et plus profondément, cet effort de faire émerger des solidarités élargies a toutefois comme effet de faire s'étioler les bases de solidarités sociales traditionnellement plus restreintes. Les tenants du «dialogue» entre partenaires sociaux et agents de l'économie ont interprété ce résultat de leurs interventions comme «un certain affaiblissement des oppositions à caractère purement idéologique qui ont caractérisé les sociétés occidentales il y a quelques années, période où l'on a assisté à des confrontations verbales chez les étudiants, les groupes ethniques, les travailleurs " ${ }^{91}$. Il se révèle toutefois plus avisé de voir avec Alain Lipietz qu' «en fait, c'est un nouveau modèle d'industrialisation, de nouveaux modes de consommation, de nouvelles relations sociales qu'il s'agit d'inventer, au Nord comme au Sud, et nécessairement ensemble» ${ }^{92}$. Pour l'instant, le capital et ses agents politiques ont nettement orienté leurs interventions en ce sens, essayant en même temps et avec un succès certain de faire percevoir toute lutte de résistance des forces les plus sévèrement touchées comme lutte d'arrière-garde et rétrograde. C'est la position inconfortable dans laquelle se trouve actuellement placé le mouvement syndical.

90. «La F.T.Q. lance son fonds de solidarité pour la création et le maintien de l'emploi », dans Le monde ouvrier, avril 1983, 10.

91. Secrétariat permanent des conférences socio-économiques, Le bilan des conférences socio-économiques du Québec, op. cit., 94.

92. Alain Lipietz, De la nowvelle division internationale du travail à la crise du fordisme périphérique, Intervention au colloque «Problemi della reprisa europea e del nuovo ordine economico internazionale», Modène, 25-27 novembre 1982, 34. 
Dans un processus de transition comme celui dont on est présentement témoin, «il est difficile aux droits acquis de résister» ${ }^{93}$. Surtout quand le recours constant à la majorité silencieuse sert d'alibi aux changements proposés et voile les intérêts ainsi servis: "Tous les systèmes actuels fonctionnent sur cette entité nébuleuse, sur cette substance flottante dont l'existence n'est plus sociale, mais statistique, et dont le seul mode d'apparition est celui du sondage » ${ }^{94}$. Mais plutôt que d'imaginer derrière le processus d'homogénéisation apparente et momentanée des sociétés un "trou noir où le social s'engouffre" ${ }^{95}$, il semble justifié de voir poindre à l'horizon une organisation renouvelée des relations sociales au sein de laquelle le travail ne sera pas disparu.

Le syndicalisme, forme organisée du travail, subira des transformations se greffant à la redéfinition actuellement en cours du travail dans la mesure même où sa capacité de résistance se révèle grandement affaiblie. L'évocation d'une telle perspective ne justifie pas pour autant les anathèmes sur le peu d'adaptabilité de l'organisation syndicale alors que le travail en général subit des attaques rappelant celles qui avaient accompagné les bouleversements majeurs qui ont historiquement marqué l'organisation du travail social. Au bout du compte, syndiqués ou pas, peu de travailleurs se trouvent à l'abri de la politique de «redéploiement du travail» dont certains éléments viennent d'être retracés, et beaucoup se trouvent profondément tiraillés entre la résistance ou la collaboration. Il demeure grandement présomptueux de soutenir que tout est joué, dans un sens ou dans l'autre.

93. Lewis A. Coser, Les fonctions du conflit social, Coll. Sociologies, Presses universitaires de France, Paris, 1982 (1956-1967), 95.

94. Jean Baudrillard, $A$ l'ombre des majorités silencieuses ou la fin du social, Bibliothèque Médiations, Denoël/Gonthier, Paris 1982 (1978), 25.

95. Ibid., 9. 